\title{
Color Attenuation Prior (CAP) for Single Image Dehazing
}

\author{
Miss. Anjana Navale ${ }^{1}$, Prof. Namdev Sawant ${ }^{2}$ and Prof. Umaji Bagal ${ }^{3}$ \\ ${ }^{1,2,3}$ Department of Computer Science and Engineering, korti, \\ Pandharpur, 413304, MS, India
}

\begin{abstract}
:
Single image haze removal has been a challenging problem due to its ill-posed nature. In this paper, we have used a simple but powerful color attenuation prior for haze removal from a single input hazy image. By creating a linear model for modeling the scene depth of the hazy image under this novel prior and learning the parameters of the model with a supervised learning method, the depth information can be well recovered. With the depth map of the hazy image, we can easily estimate the transmission and restore the scene radiance via the atmospheric scattering model, and thus effectively remove the haze from a single image. Experimental results show that the proposed approach outperforms state-of-the-art haze removal algorithms
\end{abstract} in terms of both efficiency and the dehazing effect. Keywords: Dehazing, Depth, Color attenuation prior

\section{Introduction}

Outdoor images taken in bad weather conditions (e.g., foggy or hazy) generally lose contrast and fidelity, resulting from the fact that light is absorbed and scattered by the cloudy medium such as particles and water droplets in the atmosphere through the process of propagation. Reinstatement of images taken in these specific situations has caught increasing attention in the last years. This job is important in a number of outdoor applications such as remote sensing, intelligent vehicles, object recognition and surveillance. In remote sensing systems, the recorded bands of reflected light are processed [1], [2] in order to restore the outputs. Multi-image techniques [3] solve the image de hazing difficulty by processing several input images that have been taken in different atmospheric situation. A more difficult problem is when only a single degraded image is available. Solutions for such cases have been introduced only in recent times [6]-[10].

In this paper we introduce an alternative singleimage based approach that is able to accurately dehaze images using only the original degraded information. An extended conceptual of the core idea has been recently introduced by the authors in [11]. Moreover, most automatic systems, which strongly depend on the meaning of the input images, fail to work usually caused by the degraded images.

Therefore, improved techniques of image haze removal will benefit many image understanding and computer vision applications such as aerial imagery [1], image classification [2]-[5],image/video retrieval [6]-[8], remote sensing [9]-[11] and video analysis and recognition [12]-[14].Since concentration of the haze is different from place to place and it is difficult to detect in a hazy image, image dehazing is thus a challenging task. Although Tan's approach is able to attain notable results, it tends to produce over-saturated images. Fattal [15] proposes to remove the haze from color images based on Independent Component Analysis (ICA), but the approach is time-consuming and cannot be used for grayscale image dehazing. First, this approach performs an effective per-pixel calculation, different from the majority of the earlier methods [6]-[8] that process patches. An appropriate perpixel strategy reduces the amount of artifacts, since patch based methods have some limitations due to the assumption of constant air light in every patch. In broad, the assumptions made by patch-based techniques do not hold, and therefore additional post processing steps are necessary (e.g. the method of He et al. [8] needs to smooth the transmission map by alpha-matting). Secondly, since do not estimate the depth (transmission) map, the difficulty of this approach is lower than most of the earlier strategies. Finally, this technique performs faster which makes it appropriate for real-time applications. 
In this paper, we propose a novel color attenuation prior to single image dehazing. This simple and powerful prior can help to create a linear model for the scene depth of the hazy image. The linear model is used to learning the parameters with a supervised learning method, the bridge between the hazy image and its corresponding depth map is built effectively. With the recovered depth information, we can easily remove the haze from a single hazy image. An overview of the proposed dehazing. The efficiency of this hazing method is high and the dehazing effectiveness is also high to that of prevailing dehazing algorithms. An overview of the proposed dehazing method is shown in Figure 1.

\section{Atmospheric Scattering Model :}

To explain the configuration of a hazy image, the atmospheric scattering model, which is proposed by McCartney in 1976 [20], is generally used in computer vision and image processing. Narasimhan and Nayar [21], [22], [23], [24] later they derive the model, and the model can be expressed as follows:

$$
\mathrm{I}(x)=J(x) t(x)+A(1-t(x))
$$

$$
t(x)=e-\beta \mathrm{d}(x)
$$

Where $\mathrm{x}$ is the position of the pixel within the image, $\mathrm{I}$ is the hazy image, $\mathrm{J}$ is the scene radiance indicating the haze-free image, $\mathrm{A}$ is the atmospheric light, $\mathrm{t}$ is the medium transmission,

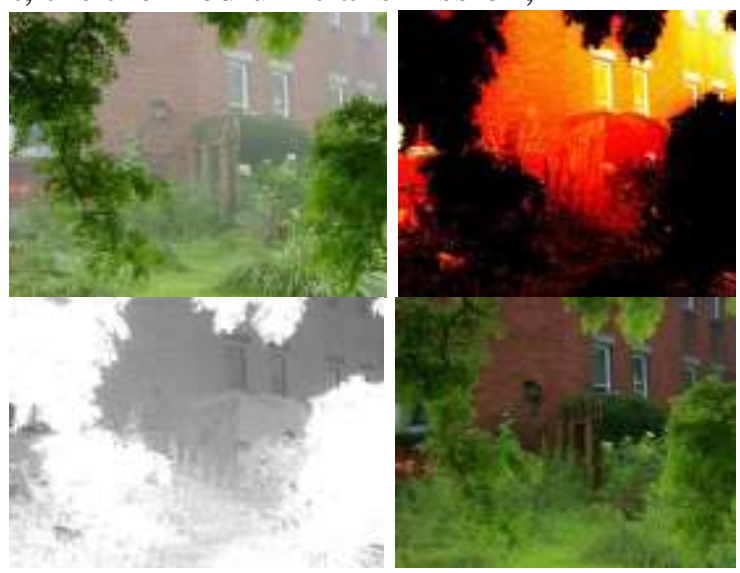

Figure 1. An overview of the proposed dehazing method. Top-left: Input hazy image. Top-right: Restored depth map. Bottom-left: Restored transmission map. Bottom-right: Dehazed image

$\beta$ is the scattering coefficient of the atmosphere and $\mathrm{d}$ is the depth of a scene. I, J and A are all 3-D vectors in RGB space. Since $I$ is known, the objective of dehazing is to estimate $A$ and $t$, then restore $\mathbf{J}$ according to Equation (1). It is significance noting that the depth of scene $d$ is the most important information. Since the scattering coefficient $\beta$ can be regarded as a constant in homogeneous atmosphere condition [23], the average transmission $t$ can be approximated easily according to Equation (2) if the depth of the scene is known. Moreover, in the ideal case, the range of $\mathrm{d}(\mathrm{x})$ is $[0,+\infty)$ as the scenery objects that show in the image can be very far away from the spectator, and we have:
$\mathrm{I}(x)$
$=$
A,
$d(x)$
$\infty$
(3)

Equation (3) shows that the intensity of the pixel, which makes the depth tend to infinity, be able to stand for the value of the atmospheric light A. Note that, if $d(x)$ is large enough, $t(x)$ tends to be very small according to Equation (2), and I(x) equals A approximately. Therefore, instead of calculating the atmospheric light A by Equation (3), we can approximation $\mathrm{A}$ by the following equation given a threshold $d_{\text {thresold }}$ :

$\mathrm{d}(x)=A,(x) \geq d_{\text {thresold }}$

We also see the fact that it is not hard to satisfy this constraint: $\mathrm{d}(\mathrm{x})>d_{\text {thresold. }}$ In most cases, a hazy image taken outdoor has a far-away view that is kilometers away from the observer. In other words, the pixel belonging to the area with a far-away view in the image should have a very large depth $d_{\text {thresoid }}$. Assuming that every hazy image has a distant view, we have:

$$
\mathrm{d}(x) \geq d \text { thresold, } x \in\{\mid \forall y: d(y) \leq d(x)\}
$$

Based on this hypothesis, the atmospheric light $\mathrm{A}$ is given by:

$$
A=I(x), x \in\{x \mid \forall y: d(y) \leq d(x)\}
$$

On this condition, the job of dehazing can be further changed into depth information restoration. However, it is also a demanding task to obtain the depth map from a single hazy image. In the next part, present a novel color attenuation prior which is useful for restoring the depth information from a single hazy image directly.

\section{Color Attenuation Prior}

As very small information about the scene structure is available it is very difficult to detect or remove the haze from a distinct image in computer vision, In spite of this, the human brain can quickly recognize the hazy area from the natural scenery without any extra information. This motivated us to conduct a 
large number of experiments on a variety of hazy images to find the information and seek a new prior for single image dehazing. Interestingly, find that the brightness and the saturation of pixels in a hazy image vary sharply along with the change of the haze attention. It seems that the three properties (the brightness, the saturation, and the difference) are prone to vary frequently in a sole hazy image according to this observation. Is this coincidence, or is there a fundamental reason behind this? To find the answer this question, first, re-examine the process of imaging. In the haze-free location, the scene element reflects the energy that is from the lighting source (e.g., direct sunlight, diffuse skylight, and light reflected by the ground), lost a portion of energy when it reaches the imaging system. The imaging system collects the inward energy reflected from the scene part and focuses it on the image plane. Without the effect of haze, outdoor images are typically are of different colors. In hazy conditions, in contrast, the situation becomes more difficult. There are two mechanisms (the direct attenuation and the air light) in imaging under hazy conditions [22]. On one hand, the direct attenuation caused by the decrease in reflected energy leads to the low intensity of the brightness. To recognize this, review the atmospheric scattering method. The term $\mathrm{J}(\mathrm{x}) \mathrm{t}(\mathrm{x})$ in Equation (1) is used for describing the direct attenuation.

It reveals the fact that the intensity of the pixels within the image will reduce in a multiplicative way. So it turns out that the brightness tends to decrease under the control of the direct attenuation. On the other hand, the white or gray air light, which is produced by the scattering of the environmental illumination, enhances the brightness and minimizes the saturation. This can also be explained by the atmospheric scatter model. The rightmost term A $(1-t(x))$ in Equation (1) represents the effect of the air light. It can be deduced from this term that the effect of the white or gray air light on the experiential values is additive. Thus, caused by the air light, the brightness is increased while the saturation is decreased. Since the air light plays a more important role in most cases, hazy areas in the image are categorized by high brightness and low saturation. The denser the haze is, the stronger the influence of the air light would be. Since the concentration of the haze increases along with the change of the scene depth in general, make an assumption that the depth of the scene is positively correlated with the concentration of the haze and it gives: $\mathrm{d}(x) \propto c(x) \propto v(x)-s(x)$

Where $\mathrm{d}$ is the scene depth, $\mathrm{c}$ is the concentration of the haze, $v$ is the brightness of the scene and $s$ is the saturation. We observe these statistics as color attenuation prior. Figure 1 gives the statistical explanation of the color attenuation prior to the HSV color model. Figure 2(a) is the HSV color model, and Figure 2(b-d) are the near, moderate-distance and far scene depths, respectively. Vector I indicates the hazy image, passing through the origin and performing the projection of the vector I onto a horizontal plane Setting the angle between vector I and its projection as $\alpha$, according to the HSV color model, when $\alpha$ varies between 0 and 90 degrees, the higher the value of $\alpha$ is, the higher the value of tangent $\alpha$ is, which indicates the greater the difference between the component of $\mathrm{I}$ in the direction of $\mathrm{V}$ and the component of $\mathrm{I}$ in the direction of $\mathrm{S}$. As the depth increases, the value $\mathrm{v}$ increases and the saturation $\mathrm{s}$ decreases, and therefore $\alpha$ increases. In other words, the angle $\alpha$ is positively correlated with the depth. It is worth to point out that Equation (7) is just an intuitional result of the observation and it cannot be an accurate expression about the links among $\mathrm{d}, \mathrm{v}$, and $\mathrm{s}$. We will find the way to create a more robust expression in the following sections. (a)

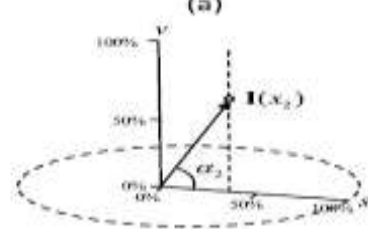

(c)

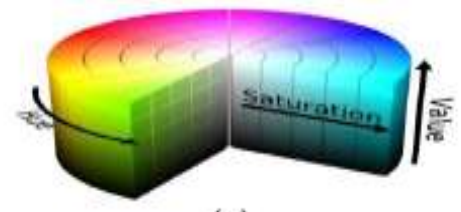

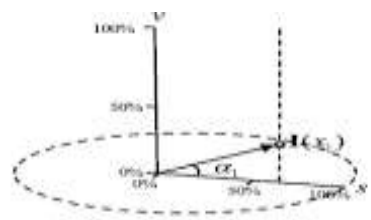

(b)

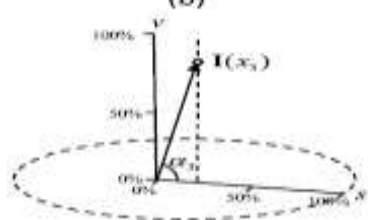

(d)
Figure. 2. The geometric description of the color attenuation prior. (a) The HSV color model. (b) The near scene depth condition. (c) The moderate distance condition. (d) The far scene depth condition

\section{Scene Depth Restoration}

\section{A. The Linear Model Definition}

As the disparity between the brightness and the saturation can approximately represent the concentration of the haze, then it can create a linear model, i.e., a more precise expression, as follows:

$$
\mathrm{d}(x)=\theta_{0}+\theta_{1} v(x)+\theta_{0} s(x)+\varepsilon(x)
$$


Where $x$ is the position within the image, $d$ is the scene depth, $v$ is the brightness component of the hazy image, $s$ is the saturation component, $\theta 0, \theta 1, \theta 2$ are the unknown linear coefficients, $\varepsilon(x)$ is a random variable expressive the random error of the model, and $\varepsilon$ can be regarded as a random image. Use a Gaussian density for $\varepsilon$ with zero mean and variable $\sigma 2$ (i.e. $\varepsilon(x) \sim N(0, \sigma 2)$ ). According to the property of the Gaussian distribution:

$$
\mathrm{d}(x) \sim\left(d(x) \mid x, \theta_{0}, \theta_{1}, \theta_{2}, \sigma^{2}\right)=\left(\theta_{0}+\theta_{1} v+\theta_{2} s, \sigma^{2}\right)
$$

One of the most important advantages of this model is that it has the edge-preserving property. To show this, calculate the gradient of $d$ in Equation (8):

$$
\nabla d=\theta_{1} \nabla v+\theta_{2} \nabla_{s}+\nabla_{\varepsilon}
$$

Due to that $\sigma$ can never be too large in practice; the value of $\varepsilon(x)$ tends to be very low and close to zero. In this case, the value of $\nabla_{\varepsilon}$ is low enough to be ignored.

This further ensures that the depth information can be well recovered even near the depth discontinuities in the scene. In the following sections, use a simple and efficient supervised learning method to determine the coefficients $\theta 0, \theta 1, \theta 2$ and the variable $\sigma 2$.

\section{B. Training Data Collection}

In order to discover the coefficients $\theta 0, \theta 1$, and $\theta 2$ exactly, the training data are needed. In this case, a training sample consists of a hazy image and its corresponding ground fact depth map. Unfortunately, the depth map is very complex to obtain due to the fact that there are no reliable means to calculate the depths of outdoor scenes. Current depth cameras such as Kinect are not able to obtain the accurate depth information. Inspired by

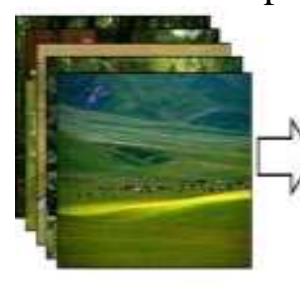

The haze-free images

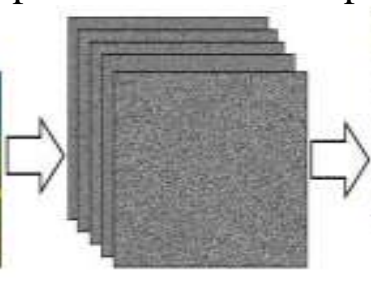

The generated random depth maps

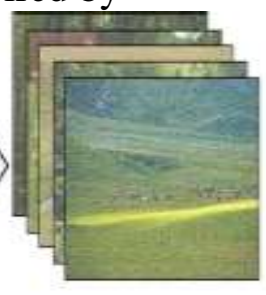

The generated hazy images
Figure 3. The process of generating the training samples with the haze-free images. Left sub-figure: The haze-free images. Center sub-figure: The generated random depth maps. Right sub-figure: The generated hazy images.
Tang et al.'s method for preparing the training data [27], then collect the haze-free images from Google Images and Flickr and use them to create the synthetic depth maps and the equivalent hazy images for obtaining sufficient training samples. Generating the training samples is illustrated in Figure 3. Firstly, we generate a random depth map of each haze-free image with the same size. The values of the pixels within the synthetic depth map are drawn from the standard uniform distribution on the open interval $(0,1)$. Secondly, we generate the random atmospheric light $\mathrm{A}(\mathrm{k}, \mathrm{k}, \mathrm{k})$ where the value of $\mathrm{k}$ is between 0.85 and 1.0. Finally, we generate the hazy image I with the random depth map $\mathrm{d}$ and the random atmospheric light $\mathrm{A}$ according to Equation (1) and Equation (2).

\section{Learning Strategy}

In the joint conditional concentration:

$L=(\mathrm{d}(x 1), \ldots, d(x n) \mid x 1, \ldots, x n, \theta 0, \theta 1, \theta 2, \sigma 2)$

Where $\mathrm{n}$ is the total number of pixels within the training hazy images, $(x n)$ is the depth of the nth scene point, and $\mathrm{L}$ is the likelihood. Let that the random error at each scene point is independent (i.e. $(\varepsilon 1, \ldots, \varepsilon n)=\Pi i=1, \ldots n p(\varepsilon i)) \rrbracket$, it can rewrite Equation (11) as:

\section{$L=\prod_{i=1}^{n} p\left(d\left(x_{i}\right) \mid x_{i}, \theta_{0}, \theta_{1}, \theta_{2}, \sigma_{2}\right)$}

According to Equation (9) and Equation (12), it gives:

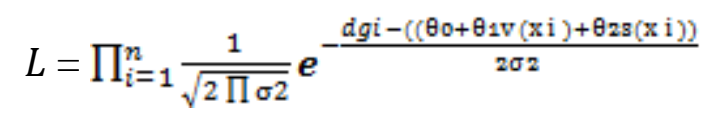

where $d g i$ represents the ground truth depth of the ith scene point. So the problem is to find the optimal values of $\theta 0, \theta 1, \theta 2$, and $\sigma$ to maximum $L$. For convenience, instead of maximizing the probability directly, we maximize the natural logarithm of the probability $\ln \mathrm{L}$. Therefore, the problem can be expressed as follows:

$$
\underset{\theta 0, \theta 1, \theta 2, \sigma}{\arg \max } \ln \mathrm{L}=\sum_{i=1}^{n} \ln \left(\frac{1}{\sqrt{2 \prod \sigma 2}} e^{-\frac{\operatorname{dgi}\left(-\left(\theta_{0}+\theta 1 v(\mathrm{xi})+\theta 2 \mathrm{~g}(\mathrm{xi})\right)\right.}{\mathrm{n} \sigma \mathrm{z}}}\right.
$$

To solve the problem, first calculate the partial derivative of

$\operatorname{lnL}$ with respect to $\sigma$ and make it equal to zero: 
$\frac{\partial \ln \mathrm{L}}{\partial \sigma}=-\frac{n}{\sigma}+\frac{1}{\sigma 3} \sum_{i=1}^{n}(\operatorname{dgi}-(\theta 0+\theta 1 \mathrm{v}(\mathrm{xi})+\theta 2 \mathrm{~s}(\mathrm{xi})))$

(15)

According to Equation (18), the maximum likelihood estimate for the variable $\sigma 2$ is:

$\sigma^{2}=\frac{1}{n} \sum_{i=1}^{n}(d g i-(\operatorname{dgi}-(\theta 0+\theta 1 \mathrm{v}(\mathrm{xi})+\theta 2 \mathrm{~s}(\mathrm{xi})))$

(16)

As for the linear coefficients $\theta 0, \theta 1$ and $\theta 2$ use the gradient descent algorithm to estimate their values. By taking the partial derivatives of $\operatorname{lnL}$ with respect to $\theta 0, \theta 1$ and $\theta 2$ respectively,

Algorithm 1 Parameters Estimation

Input: the training brightness vector $\mathrm{v}$, the training saturation vector $\mathrm{s}$, the training depth vector $\mathrm{d}$, and the number of iterations $t$

Output: linear coefficient $\theta 0, \theta 1, \theta 2$, the variance $\sigma 2$

\section{Auxiliary functions:}

Function for obtaining the size of the vector: $n=$ size (in)

Function for calculating the square: out=square (in)

\section{Begin}

1: $\mathrm{n}=\operatorname{size}(\mathrm{v})$;

2: $\theta 0=0 ; \theta 1=1 ; \theta 2=-1$;

$3:$ sum $=0 ;$ wSum $=0 ;$ vSum $=0 ;$ sSum $=0$;

4: for iteration from 1 to $t$ do

5: for index from 1 to $\mathrm{n}$ do

6: temp $=[i]-\theta 0-\theta 1 * v[i]-\theta 2 * s$;

7: $w$ Sum $=$ vSum + temp;

8: $s$ Sum $=$ sSum $+[i] *$ temp;

9: $s$ Sum $=$ sSum $+[i] *$ temp;

10: sum $=$ sum $+($ temp $)$;

11: end for

12: $\sigma 2=\operatorname{sum} / n$;

13: $\theta 0=\theta 0+$ wSum ; $\theta 1=\theta 1$ vSum; $\theta 2=\theta 2+$ sSum;

\section{4: end for}

\section{End}

It can obtain the following expressions:

$$
\begin{aligned}
& \frac{\partial \ln \mathrm{L}}{\partial \sigma}=\frac{1}{\sigma 2} \sum_{i=1}^{n}(d g i-((\theta 0+\theta 1 v(\mathrm{xi})+\theta 2 \mathrm{~s}(\mathrm{xi}))) \\
& \frac{\partial \ln \mathrm{L}}{\partial \sigma}=\frac{1}{\sigma 2} \sum_{i=1}^{n} v(x i)(d g i-((\theta 0+\theta 1 v(\mathrm{xi})+\theta 2 s(\mathrm{xi}))) \\
& \frac{\partial \ln \mathrm{L}}{\partial \sigma}=\frac{1}{\sigma 2} \sum_{i=1}^{n} s(x i)(\operatorname{dg} i-((\theta 0+\theta 1 v(\mathrm{xi})+\theta 2 \mathrm{~s}(\mathrm{xi})))
\end{aligned}
$$

The expression for updating the linear coefficients can be in brief expressed by:

$\theta i=\theta i+\frac{\partial \ln \mathrm{L}}{\partial \theta \mathrm{i}}$ s.t. $i \epsilon\{0,1,2\}$

It is worth noting that the expression above is used for iterating dynamically, and the notation: = does not express the mathematical equality but means that setting the value of $\theta \mathrm{i}$ in the left term to be the value of the right term. The process for learning the linear coefficients $\theta 0, \theta 1, \theta 2$ and the variable $\sigma 2$ is shown in Algorithm 1.

\section{Estimation of the Depth Information}

As the among link between the scene depth d, the brightness $\mathrm{v}$, and the saturation $\mathrm{s}$ has been recognized and the coefficients have been estimated, then it can restore the depth map of a given input hazy image according to Equation (8).However, this model may fail to work in some particular situations. For example, the white objects in an image are frequently with high values of the brightness and low values of the saturation. Therefore, the model tends to consider the scene objects with white color as being far away. Unluckily, this misclassification will result in inaccurate estimation of the depth in some cases. In the white geese in the image are the regions for which the model can barely handle, and these regions are wrongly estimated with high depth values in the depth map. To overcome this problem, need to consider each pixel in the neighborhood. Based on the hypothesis that the scene depth is locally constant, and then process the raw depth map by:

$$
d_{r}(x)=\min _{y \in \Omega r(x)} d(y)
$$

Where $r(x)$ is an $r \times r$ neighborhood centered at $x$, and $d r$ is the depth map with scale $r$. The new depth map $d 15$ can well handle the geese regions. However, it is also obvious that the blocking artifacts show in the image. To process the depth map, use the guided image filtering [28]to smooth the image. The final restored depth map of the hazy image can be seen, the blocking artifacts.

\section{Scene Radiance Recovery}

\section{A. Estimation of the Atmospheric Light :}

The main idea of estimating the atmospheric light explained in Section II. In this section, explain the method in more detail. As the depth map of the input hazy image has been improved, the allocation of the scene depth is known. Bright regions in the map 
stand for distant places. According to Equation (6), pick the top 0.1 percent brightest pixels in the depth map, and select the pixel with maximum intensity in the corresponding hazy image I among these brightest pixels as the atmospheric light A.

\section{B. Scene Radiance Recovery :}

Now that the depth of scene $d$ and the atmospheric light Aare known, it can estimate the medium transmission t simply according to Equation (2) and improve the scene radiance Jin Equation (1). For ease, rewrite Equation (1) as follows:

$$
J(x)=\quad \frac{I(x)-A}{t(x)}+A=\frac{I(x)-A}{e^{-\beta d(x)}}+A
$$

For avoiding producing too much noise, limit the value of the transmission $\mathrm{t}(\mathrm{x})$ between 0.1 and 0.9 . So the final function used for restoring the scene radiance $\mathrm{J}$ in the method can be expressed by:

$$
J(x)=\frac{I(x)-A}{\min \left[\max \left\{e^{-\beta d(x)}, 0.1\right\}, 0.9\right\}}+A
$$

Where $\mathbf{J}$ is the haze-free image. Figures (4) show some finishing results of dehazing of the given method. Note that the scattering coefficient $\beta$, which can be regarded as a constant [23] in homogeneous regions, the ability of a unit volume of atmosphere to scatter light in all directions. In other words, $\beta$ determines the intensity of dehazing indirectly. Therefore, a moderate $\beta$ is essential when dealing with the images with dense-haze regions. In most cases, $\beta=1.0$ is more than enough.

\section{Experiments}

In order to verify the usefulness of the given dehazing method, test it on various hazy images and compare with $\mathrm{He}$ et al.'s [17] method. All the algorithms are implemented in the MatlabR2012a environment on a $\mathrm{P} 4-2.3 \mathrm{GHz}$ PC with 4GB RAM. The parameters used in the given method are initialized as follows: $r=15, \beta=1.0, \theta 0=0.121779$,

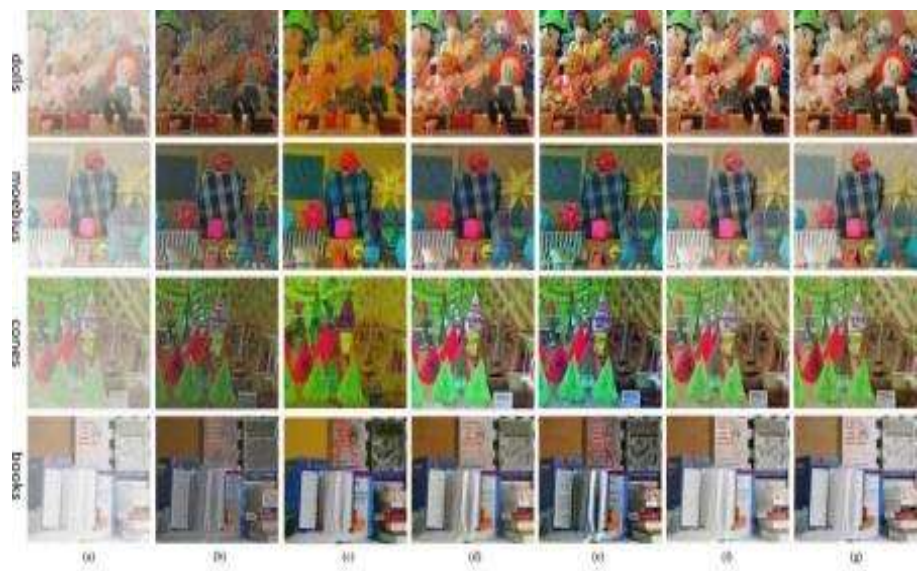

Figure. 4. Qualitative comparison of different methods on real-world images (a) The hazy images (b) Tarel et al.'s results (c) Nishino et al.'s results(d) He et al.'s results (e) Meng et al.'s results (f) multiscale methods result (g) Our results

Sobel detection refers to computing the gradient magnitude of an image using $3 \times 3$ filters. Where "gradient magnitude" is, for each a pixel, a number giving the greatest rate of change in light intensity in the direction where intensity is changing fastest.

Canny edge detection goes a bit further by removing speckle noise with a low pass filter first, then applying a Sobel filter, and then doing nonmaximum suppression to pick out the best pixel for edges when there are multiple possibilities in a local neighborhood. That's a simplification, but basically its smarter than just applying a threshold to a Sobel filter, but it is still fairly low level processing.

Edge-preserving smoothing is an image processing technique that smooth's away textures whilst retaining sharp edges. E.g. the bilateral filter, the guided filter. When we need to preserve edge information and at the same time preserve the edges. Even when uniform smoothing does not remove the boundaries, it does distort them. This is not acceptable in the context of, for example, medical imaging.

Typical edge preserved filtering includes two types: Kuwahara filtering and selective mask filtering. The basic process of them is described as follows: Firstly, some different templates are made based on the center pixel. Secondly, the mean value and the standard deviation of the pixels in different templates are calculated. Finally, the gray value of the center pixel is defined as the mean value in the template where the standard deviation is the least.

The Kuwahara filter is a non-linear smoothing filter used in image processing for 
adaptive noise reduction. Most filters that are used for image smoothing are linear low-pass filters that effectively reduce noise but also blur out the edges. However the Kuwahara filter is able to apply smoothing on the image while preserving the edges.

\section{Conclusion}

In order to reduce Gaussian noise, we usually want to smooth the image. But we do not want to smooth out the true edges in the image. Hence, we use Edge Preserving Smoothing filter. In this paper, we have used "Kuwahara Filter" instead of "Guided Filter". [He et.al]. Guided filters use integral image to perform smoothing. Hence, the computational complexity is not dependent on the size of the smoothing kernel. The computational time depends may increase if size of filter image increase. But Kuwahara filter does not require filter image and it is a non-linear smoothing filter.

Although we have found a way to model the scene depth with the brightness and the saturation of the hazy image, there is still a common problem to be solved. That is, the scattering coefficient $\beta$ in the atmospheric scattering model cannot be regarded as a constant in inhomogeneous atmosphere conditions. For example, a region which is kilometres away from the observer should have a very low value of $\beta$. Therefore, the dehazing algorithms which are based on the atmospheric scattering model are prone to underestimating the transmission in some cases. As almost all the existing single image dehazing algorithms are based on the constant- $\beta$ assumption, a more flexible model is highly desired. To overcome this challenge, some more advanced physical models can be taken into account. In future work these methods may be tested on videos.

\section{References}

[1] G. A. Woodell, D. J. Jobson, Z.-U. Rahman, and G. Hines, "Advanced image processing of aerial imagery," Proc. SPIE, vol. 6246, p. $62460 \mathrm{E}$, May 2006.

[2] L. Shao, L. Liu, and X. Li, "Feature learning for image classification viamulti objective genetic programming," IEEE Trans. Neural Netw. Learn.Syst., vol. 25, no. 7, pp. 1359-1371, Jul. 2014.

[3] F. Zhu and L. Shao, "Weakly-supervised cross-domain dictionary learning for visual recognition," Int. J. Comput. Vis., vol. 109, nos. 1-2,pp. 42-59, Aug. 2014.

[4] Y. Luo, T. Liu, D. Tao, and C. Xu, "Decomposition-based transfer distance metric learning for image classification,"
IEEE Trans. ImageProcess., vol. 23, no. 9, pp. 3789-3801, Sep. 2014.

[5] Tao, X. Li, X. Wu, and S. J. Maybank, "Geometric mean for subspaceselection," IEEE Trans. Pattern Anal. Mach. Intell., vol. 31, no. 2,pp. 260-274, Feb. 2009.

[6] J. Han et al., "Representing and retrieving video shots in human-centricbrain imaging space," IEEE Trans. Image Process., vol. 22, no. 7,pp. 2723-2736, Jul. 2013.

[7] J. Han, K. Ngan, M. Li, and H.-J. Zhang, "A memory learning frameworkfor effective image retrieval," IEEE Trans. Image Process., vol. 14,no. 4, pp. 511-524, Apr. 2005.

[8] Tao, X. Tang, X. Li, and X. Wu, "Asymmetric bagging and randomsubspace for support vector machines-based relevance feedback in imageretrieval," IEEE Trans. Pattern Anal. Mach. Intell., vol. 28, no. 7,pp. 1088-1099, Jul. 2006.

[9] J. Han, D. Zhang, G. Cheng, L. Guo, and J. Ren, "Object detection inoptical remote sensing images based on weakly supervised learning andhigh-level feature learning," IEEE Trans. Geosci. Remote Sens., vol. 53,no. 6, pp. 3325-3337, Jun. 2015.

[10] Cheng et al., "Object detection in remote sensing imagery using adiscriminatively trained mixture model," ISPRS J. Photogramm. RemoteSens., vol. 85, pp. 3243, Nov. 2013.

[11] J. Han et al., "Efficient, simultaneous detection of multi-class geospatialtargets based on visual saliency modeling and discriminative learning ofsparse coding," ISPRS J. Photogramm. Remote Sens., vol. 89, pp. 37-48, Mar. 2014.

[12] L. Liu and L. Shao, "Learning discriminative representations fromRGB-D video data," in Proc. Int. Joint Conf. Artif. Intell., Beijing, China,2013, pp. 1493- 1500.

[13] D. Tao, X. Li, X. Wu, and S. J. Maybank, "General tensor discriminant analysis and Gabor features for gait recognition," IEEE Trans. PatternAnal. Mach. Intell., vol. 29, no. 10, pp. 1700-1715, Oct. 2007.

[14] Z. Zhang and D. Tao, "Slow feature analysis for human actionrecognition," IEEE Trans. Pattern Anal. Mach. Intell., vol. 34, no. 3,pp. 436-450, Mar. 2012.

[15] R. Fattal, "Single image dehazing," ACM Trans. Graph., vol. 27, no. 3, p. 72, Aug. 2008. 
[16] P. S. Chavez, Jr., “An improved dark-object subtraction technique for atmospheric scattering correction of multispectral data," Remote Sens. Environ., vol. 24, no. 3, pp. 459-479, Apr. 1988.

[17] K. He, J. Sun, and X. Tang, "Single image haze removal using dark channel prior," IEEE Trans. Pattern Anal. Mach. Intell., vol. 33, no. 12, pp. 2341-2353, Dec. 2011.

[18] L. Breiman, "Random forests," Mach. Learn., vol. 45, no. 1, pp. 5-32, Oct. 2001.

[19] Q. Zhu, J. Mai, and L. Shao, "Single image dehazing using color attenuation prior," in Proc. Brit. Mach. Vis. Conf. (BMVC), Nottingham, U.K., 2014, pp. 1-10.

[20] E. J. McCartney, Optics of the Atmosphere: Scattering by Molecules and Particles. New York, NY, USA: Wiley, 1976.

[21] S. K. Nayar and S. G. Narasimhan, "Vision in bad weather," in Proc. IEEE Int. Conf. Comput. Vis. (ICCV), vol. 2. Sep. 1999, pp. 820-827.

[22] S. G. Narasimhan and S. K. Nayar, "Contrast restoration of weather degraded images," IEEE Trans. Pattern Anal. Mach. Intell., vol. 25, no. 6, pp. 713-724, Jun. 2003.

[23] S. G. Narasimhan and S. K. Nayar, "Vision and the atmosphere," Int. J. Comput. Vis., vol. 48, no. 3, pp. 233-254, Jul. 2002.

[24] S. G. Narasimhan and S. K. Nayar, "Removing weather effects from monochrome images," in Proc. IEEE Conf. Comput. Vis. PatternRecognit. (CVPR), 2001, pp. II- 186-II-193.
[25] J.-P. Tarel, N. Hautière, L. Caraffa, A. Cord, H. Halmaoui, and D. Gruyer, "Vision enhancement in homogeneous and heterogeneousfog," IEEE Intell. Transp. Syst. Mag., vol. 4, no. 2, pp. 6-20, Apr. 2012.

[26] C. O. Ancuti, C. Ancuti, C. Hermans, and P. Bekaert, "A fast semiinverse approach to detect and remove the haze from a single image," in Proc. Asian Conf. Comput. Vis. (ACCV), 2010, pp. 501-514.

[27] K. Tang, J. Yang, and J. Wang, "Investigating haze-relevant features in a learning framework for image dehazing," in Proc. IEEE Conf. Comput. Vis. Pattern Recognit. (CVPR), Jun. 2014, pp. 29953002.

[28] K. He, J. Sun, and X. Tang, "Guided image filtering," IEEE Trans. Pattern Anal. Mach. Intell., vol. 35, no. 6, pp. 1397-1409, Jun. 2013.

[29] A. J. Preetham, P. Shirley, and B. Smits, "A practical analytic model for daylight," in Proc. ACM Special Interest Group Comput.Graph. (SIGGRAPH), pp. 91-100.

[30] Q. Zhu, J. Mai and L. Shao, ”A Fast Single Image Haze Removal Algorithm Using Color Attenuation Prior," in IEEE Transactions on Image Processing, vol. 24, no. 11, pp. 35223533, Nov. 2015. 